\title{
Drug Accountability Sponsor Defined Identifier
}

National Cancer Institute

\section{Source}

National Cancer Institute. Drug Accountability Sponsor Defined Identifier. NCI Thesaurus. Code $C 87878$.

One or more sponsor defined characters used to identify, name, or characterize the drug accountability assessment. 